\title{
Growth Mode Transition Involving a Potential-Dependent Isotropic to Anisotropic Surface Atom Diffusion Change. Gold Electrodeposition on HOPG followed by STM
}

\author{
H. Martín, P. Carro, A. Hernández Creus, S. González, R. C. Salvarezza, ${ }^{\dagger}$ and \\ A. J. Arvia*, \\ Departamento de Química Física, Universidad de La Laguna, La Laguna, Tenerife, Spain, \\ and Instituto de Investigaciones Fisicoquímicas Teóricas y Aplicadas (INIFTA), Sucursal 4, \\ Casilla de Correo 16, (1900) La Plata, Argentina
}

Received July 16, 1996. In Final Form: October 14, $1996^{\otimes}$

\begin{abstract}
The electrodeposition of gold on highly oriented pyrolytic graphite (HOPG) from acid aqueous solutions was studied by using electrochemical techniques complemented with ex-situ scanning tunneling microscopy (STM). The kinetics of gold electrodeposition is consistent with a nucleation and three-dimensional growth process under diffusion control from the solution side. As the applied potential moves in the negative direction, the gold crystal density increases, and the crystal shape changes from a Euclidean to a dendritic fractal morphology. This transition can be assigned to the anisotropic surface diffusion of gold adatoms induced by the applied electric potential. A model including a potential-dependent energy barrier at step edges accounts for the morphology transition for gold electrodeposition on HOPG.
\end{abstract}

\section{Introduction}

Solid phase pattern formation and pattern selection under either homoepitaxial or heteroepitaxial nonequilibrium growth conditions have been an intriguing fields for a long time and have recently received considerable attention from both theoretical and experimental standpoints. Results from studies in this field show why the surface will be stable or unstable, show what the degree of irregularity at different scale levels will be, and provide reliable ways to characterize those patterns. ${ }^{1}$ These questions are of basic importance in understanding a large number of natural phenomena, such as viscous fingering, dielectric breakdown, crystallization, electrocrystallization, and growth of bacterial colonies, and in the development of novel materials. ${ }^{2}$

The growth of a solid phase may occur under two extreme kinetic situations, namely, either close to or far away from equilibrium conditions. In the former case, classical growth models provide a reasonable framework to describe the growth kinetics, ${ }^{3-5}$ whereas, for the latter, concepts from nonequilibrium thermodynamics and statistical physics are required for approaching a rationale of the kinetics of fast solid phase growth processes.

Phase growth out of equilibrium is still a poorly understood topic, and its comprehensive theoretical understanding is also lacking. There is, however, a good understanding of diffusion-limited aggregates at the phenomenological level, as the diffusion-limited aggregation (DLA) model accounts for the formation of many patterns in which the typical fractal structure of DLA aggregates arises from the tip-splitting instability.

\footnotetext{
* To whom correspondence should be addressed.

${ }^{\dagger}$ Instituto de Investgaciones Fisicoquímicas Teóricas y Aplicadas (INIFTA).

${ }^{\otimes}$ Abstract published in Advance ACS Abstracts, December 15, 1996.

(1) Barabasi, A. L.; Stanley, H. E. Fractal Concepts in Surface Growth; Cambridge University Press: Cambridge, 1995; and references therein.

(2) Family, F., Vicsek, T., Eds.; Dynamics of Fractal Surfaces; World Scientific: Singapore, 1991. Family, F. Physica A 1990, 168, 561 and references therein.

(3) Frank, F. C.; van der Merwe, J. H. Proc. R. Soc. London, Ser. A 1949, 198, 205.

(4) Stranski, J. N.; Krastanov, L. Ber. Akad. Wiss. Wien 1938, 146, 797.

(5) Volmer, M.; Weber, A. Z. Phys. Chem. 1926, 119, 277.
}

Metal electrodeposition appears to be an adequate experimental model system to understand the role played by both local and nonlocal effects in the growth process, particularly in the morphology of the deposit. ${ }^{6}$ Thus, by changing the applied potential, it is possible to grow surfaces from a situation close to equilibrium to nonequilibrium conditions. For the latter, depending whether the growth process is dominated by local or nonlocal effects, either a compact self-affine or an open self-similar fractal deposit can be produced. ${ }^{7}$ However, the complex growth patterns resulting from experimental systems seem to indicate that in many cases both effects contribute nonuniformly along the reaction front during the growth process. $^{8}$

Despite the advances made in recent years in modeling the surface development at growing solids, the application of models to real systems is somewhat limited because a wide variety of morphologies can usually be produced by changing the operative variables. Therefore, even under a constant set of experimental conditions, as growth proceeds, a number of morphology transitions can be observed. Several explanations for these transitions have been advanced so far, but there are still insufficient experimental data to give definite support to the various interpretations reported in the literature. ${ }^{1,2}$ In fact, when the initial stages of silver electrodeposition on platinum were followed by scanning electron microscopy on the micrometer scale, ${ }^{9,10}$ it was observed that the growth of dendrites occurred at the border of small well-defined silver crystals which were formerly produced on the substrate surface. This remarkable change in the growth mode on the micrometer scale is not surprising because changes in the growth morphologies of thin metal films resulting from electrochemical atomic layer epitaxy have been observed on various single-crystal substrates in acidic

(6) Iwamoto, A.; Yoshinobu, T.; Iwasaki, A. Phys. Rev. Lett. 1994, 72 , 4025.

(7) Kahanda, G.; Wong, P.-Z. Phys. Rev. Lett. 1992, 68, 3741

(8) Vázquez, L.; Salvarezza, R. C.; Herrasti, P.; Ocón, P.; Vara, J. M.; Arvia, A. J. Phys. Rev. 1995, B52, 2032.

(9) Hernández Creus, A.; Carro, P.; González, R.; Salvarezza, R. C.; Arvia, A. J. Electrochem. Soc. 1992, 139, 1064.

(10) Schilardi, P.; Marchiano, S. L.; Salvarezza, R. C.; Arvia, A. J. To be submitted. 

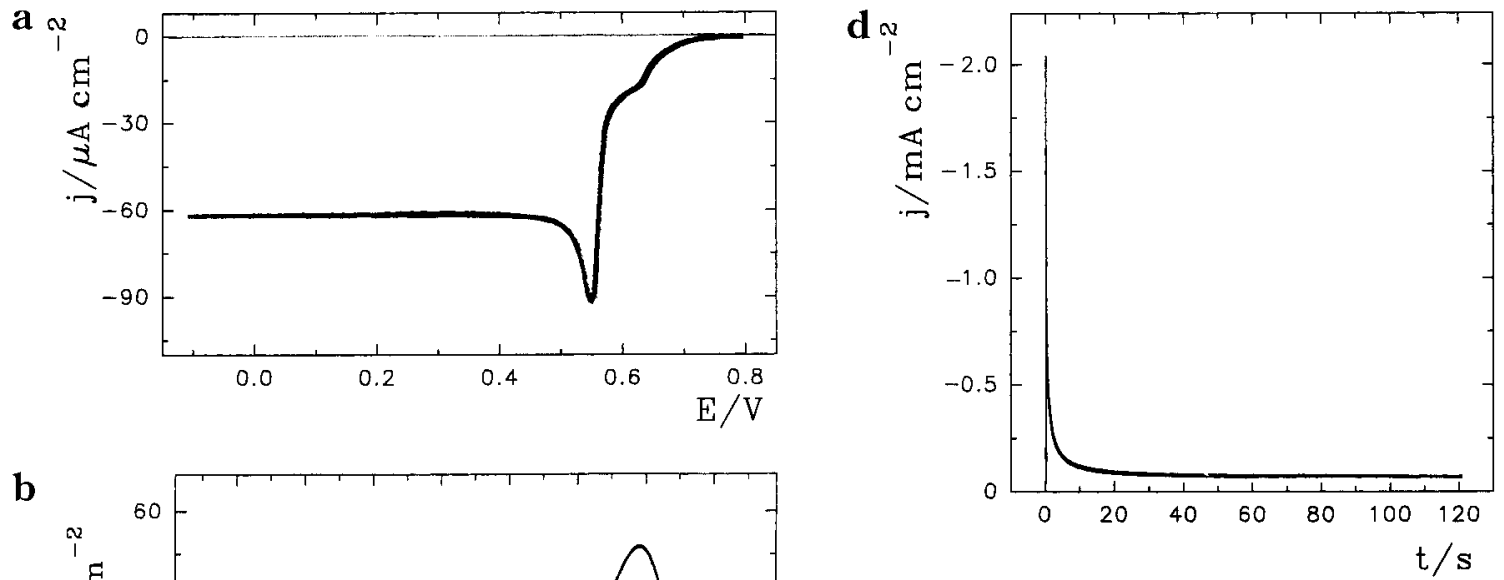

b
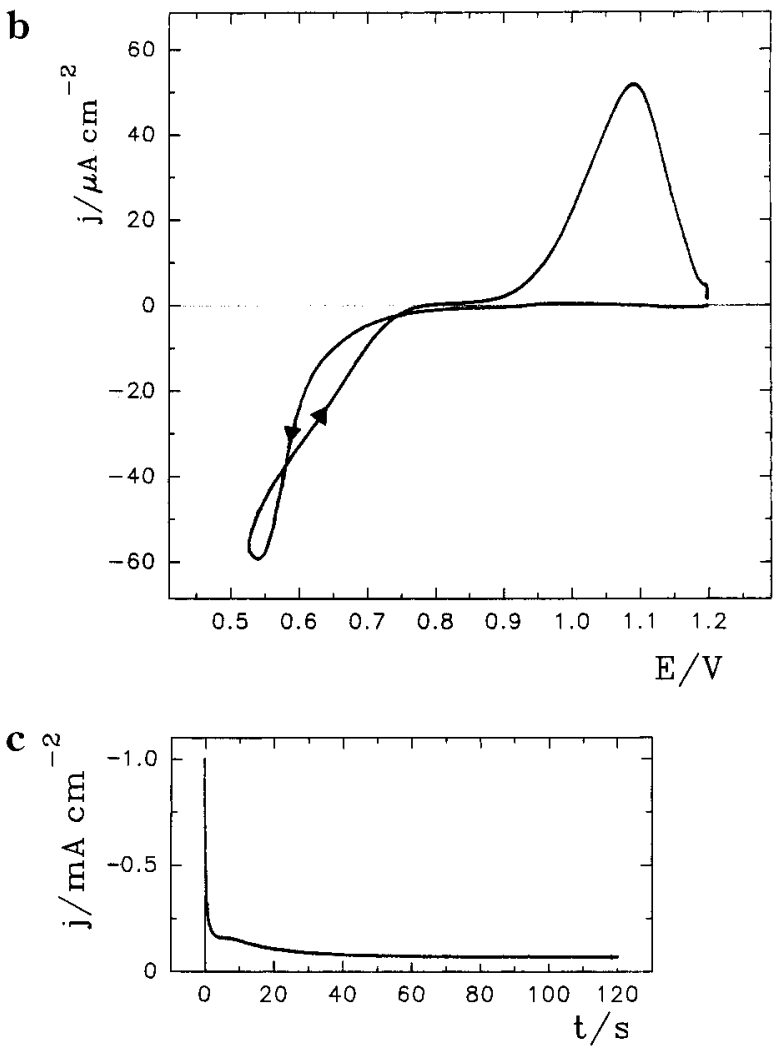

e

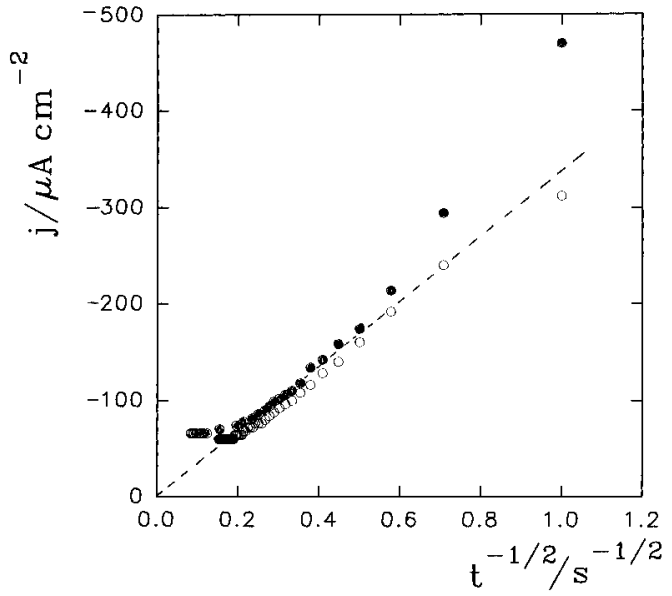

Figure 1. Electrochemical results for gold electrodeposition on $\mathrm{HOPG}$ from aqueous $5 \times 10^{-4} \mathrm{M} \mathrm{AuCl}_{3} \cdot \mathrm{HCl}+0.5 \mathrm{M} \mathrm{NaClO}_{4}+$ $10^{-2} \mathrm{M} \mathrm{HClO}_{4}$ at $25^{\circ} \mathrm{C}$. (a) Cathodic linear potential sweep polarization curve run at $V=10^{-3} \mathrm{~V} / \mathrm{s}$ between $E=0.8 \mathrm{~V}$ and $E=-0.1$ V. (b) Voltammogram run at $v=0.02 \mathrm{~V} / \mathrm{s}$ initiated toward the negative potential direction. (c) Cathodic current transient run at $E=0.54 \mathrm{~V}$. (d) Cathodic current transient run at $E=-0.10 \mathrm{~V}$. (e) Cathodic current transient data plotted as $j$ vs $t^{-1 / 2}$ : (๑) $E=$ $-0.10 \mathrm{~V}$; (O) $E=0.54 \mathrm{~V}$.

solutions. ${ }^{11,12}$ Furthermore, alternate morphology transitions between dendrites and dense branching morphology have been recently reported. ${ }^{13}$ Therefore, the selection rule in pattern formation is a complex problem for which a comprehensive description is still lacking.

In this work the electroformation of gold aggregates on highly oriented pyrolytic graphite (HOPG) in the nanometer scale range is investigated. The kinetics of gold aggregate formation in the potential range covered by this work is controlled by a nucleation and growth process under diffusion control of $\mathrm{AuCl}_{4}{ }^{-}$ions from the solution side. As the applied potential $(E)$ is moved in the negative direction, the shape of aggregates changes from Euclidean to dendritic fractal patterns, although the flux of material to the electrode surface remains constant. This transition in the growth mode can be explained in terms of an applied potential-dependent change in the energy barrier for gold

(11) Chen, C. H.; Vesecky, S.; Gewirth, A. A. J. Am. Chem. Soc. 1992 114, 451. Hachiya, T.; Itaya, K. Ultramicroscopy 1992, 42-44, 445.

(12) Ikemiya, N.; Miyaoka, N.; Hara, S. Surf. Sci. 1995, 327, 261 Ikemiya, N.; Yamada, K.; Hara, S. Surf. Sci. 1996, 348, 253.

(13) Wang, M.; Ming, N.-b. Phys. Rev. Lett. 1993, 71, 113. adatom surface diffusion at step edges, where the initially isotropic surface diffusion turns into an anisotropic surface diffusion process which stabilizes tip growth through the surface diffusion of gold adatoms in preferred step directions. The applied electric potential-dependent transition occurs in the neighborhood of the potential of zero charge of gold in the aqueous electrolyte solution and can be related to the influence of $\mathrm{Cl}^{-}$anion adsorption on gold.

\section{Experimental Section}

The gold electrodeposition on HOPG from acid solutions was studied by using conventional electrochemical techniques combined with ex-situ scanning tunneling microscopy (STM). Because of the stability of gold and HOPG in air, their morphology changes can be followed by ex-situ STM imaging.

Gold electrodeposition was made on a freshly exfoliated HOPG working electrode $\left(0.5 \mathrm{~cm}^{2}\right)$ using a conventional glass-made electrochemical cell provided with a polycrystalline platinum counterelectrode and a saturated calomel electrode (SCE) as reference electrode. Potentials in the text are referred to the SCE scale. The working solution consisted of an aqueous $5 \times$ 

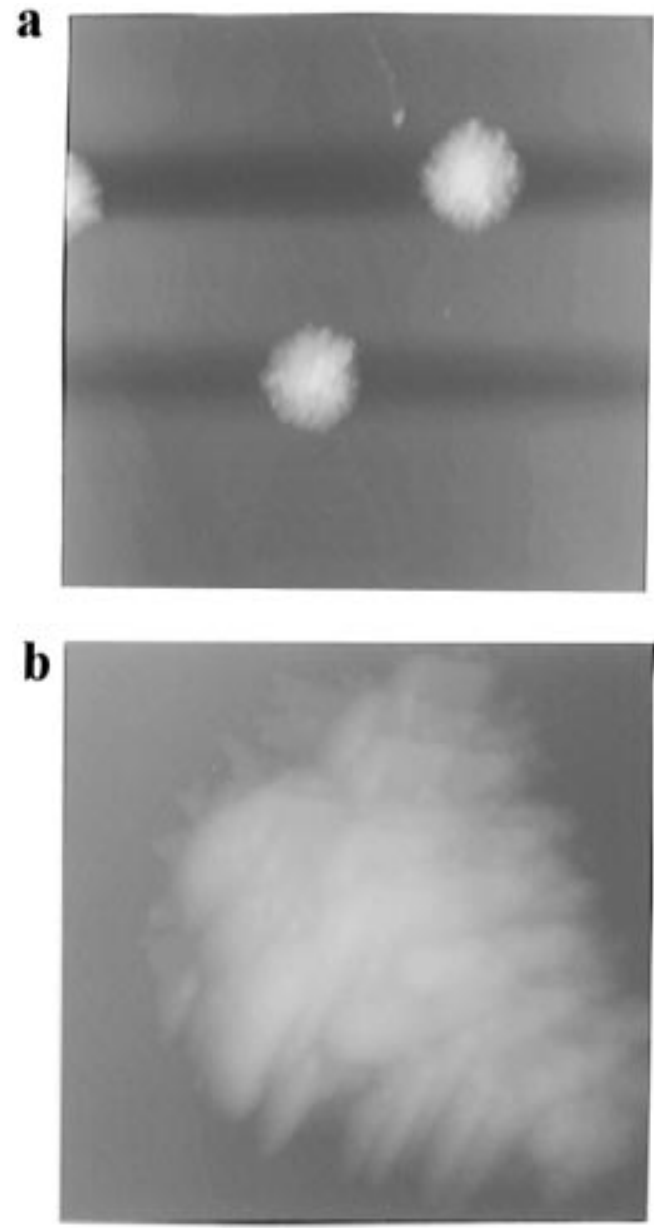

c

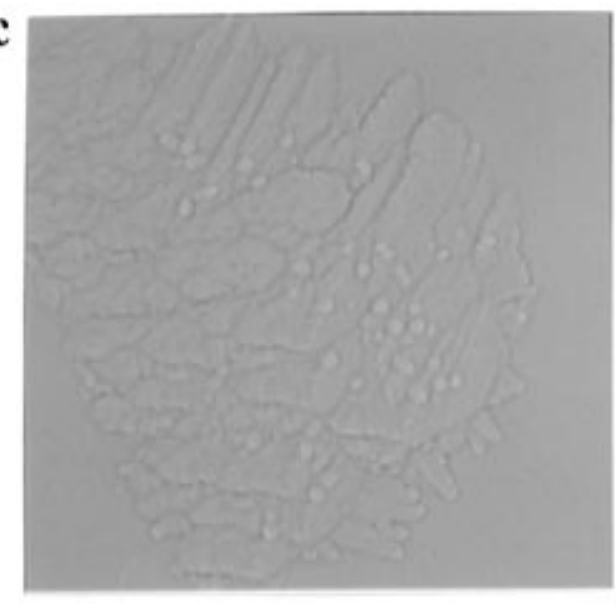

d

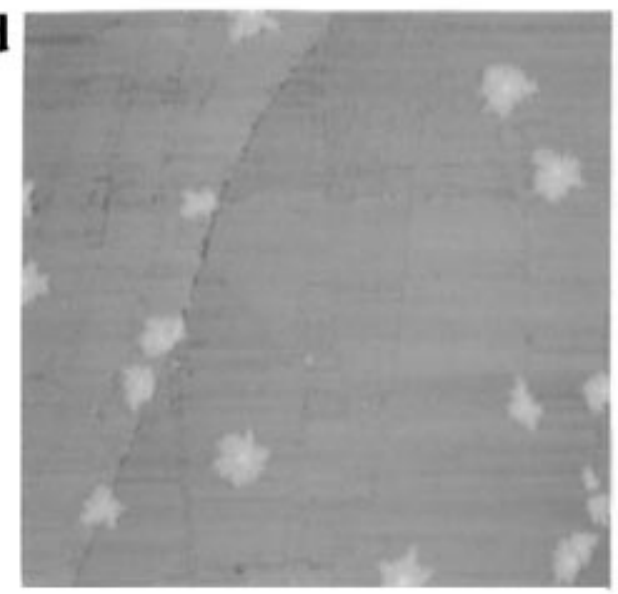

Figure 2. STM images (top view) for gold electrodeposits grown at $E>0.30 \mathrm{~V}$ at $25^{\circ} \mathrm{C}$ : (a) $10 \times 10 \mu \mathrm{m}^{2}, E=0.54 \mathrm{~V}, q=6 \mathrm{mC} / \mathrm{cm}^{2}$; (b) $1.25 \times 1.25 \mu \mathrm{m}^{2}, E=0.54 \mathrm{~V}, q=2 \mathrm{mC} / \mathrm{cm}^{2}$; (c) $1.25 \times 1.25 \mu \mathrm{m}^{2}$ high pass resulting from the STM image shown in Figure $2 \mathrm{~b}$ (Angles which are compatible with those of $\mathrm{Au}(111)$ faces can be observed.); (d) $10 \times 10 \mu \mathrm{m}^{2}, E=0.40 \mathrm{~V}, q=6 \mathrm{mC} / \mathrm{cm}^{2}$.

$10^{-4} \mathrm{M} \mathrm{AuCl}_{3} \cdot \mathrm{HCl}+0.5 \mathrm{M} \mathrm{NaClO}_{4}+1 \times 10^{-2} \mathrm{M} \mathrm{HClO}_{4}$ solution prepared from analytical reagent grade chemicals and MilliQ*Millipore water. The working solution was deaerated by bubbling purified nitrogen prior to the electrochemical runs. Experiments were performed at $25^{\circ} \mathrm{C}$.

Conventional voltammetry was run at different scan rates $(V)$, $1 \times 10^{-3} \mathrm{~V} / \mathrm{s}<V<0.1 \mathrm{~V} / \mathrm{s}$, between $1.2 \mathrm{~V}$ and $-0.1 \mathrm{~V}$, i.e. covering the range of potential where gold electrodeposition/anodic stripping conjugated processes took place on C(0001) immersed in the working solution. For this purpose, PAR equipment was employed.

Gold electrodeposits for STM imaging were produced by stepping the potential from the initial potential, $1.0 \mathrm{~V}$, to a value $E$ in the range $-0.1 \mathrm{~V}<E<0.54 \mathrm{~V}$. Electrodeposition times were in the range $1 \mathrm{~s}<t<302 \mathrm{~s}$, the charge involved being comprised in the range $0.6 \mathrm{mC} / \mathrm{cm}^{-2}<q<20 \mathrm{mC} / \mathrm{cm}^{-2}$. The value of $q$ was measured using a digitalized coulometer provided by PAR. Two different gold electrodeposits involving the same value of $q$ were made. To obtain quantitative information about the deposit growth, 30 STM images at least for each specimen covering a large number of gold nuclei were analyzed.

STM imaging was performed after the HOPG working electrode had been removed from the electrochemical cell, rinsed by successive immersions in water, and, finally, dried under nitrogen at room temperature. Immediately afterward STM images were obtained using a Nanoscope II E STM (Digital Instruments, Santa Barbara, CA) using Pt-Ir Nanotips operating in the atmosphere due to the stability of Au and HOPG surfaces in air. Images were taken at $0.5 \mathrm{nA}$ tunneling current and 0.2 $\mathrm{V}$ bias voltage. The images were taken at a low rate, usually of the order of $0.5 \mathrm{~Hz}$ for $10 \times 10 \mu \mathrm{m}^{2}$ images, to avoid either crystal damage or removal by the tip.

Gold electrodeposits remained unaltered after the STM imaging, as was demonstrated by comparing the electrodeposition charge to the anodic stripping charge measured after the STM imaging.

\section{Results}

3.1. Reference Electrochemical Data. The cathodic polarization curve for HOPG in the working solution run at $v=1 \times 10^{-3} \mathrm{~V} / \mathrm{s}$ between $E=0.8 \mathrm{~V}$ and $E=-0.1 \mathrm{~V}$ (Figure 1a) exhibits a first cathodic current density (j) when $E<E_{\mathrm{r}}=0.74 \mathrm{~V},{ }^{14} E_{\mathrm{r}}$ being the reversible potential of the $\mathrm{AuCl}_{4}{ }^{-} / \mathrm{Au}$ electrode reaction in the solution used in this work. Then, a sharp current peak at $0.54 \mathrm{~V}$ and a limiting current density $\left(j_{\mathrm{L}}\right)$ extending from $E=0.46 \mathrm{~V}$ to $E=-0.1 \mathrm{~V}$ are observed. The cathodic current corresponds to gold electrodeposition on HOPG from soluble gold species present in the working solution. As expected, the value of $j_{\mathrm{L}}$ increases with the solution stirring.

A triangular voltammetric sweep run at $v=0.02 \mathrm{~V} / \mathrm{s}$ from $E=1.2 \mathrm{~V}$ in the negative direction shows the characteristic loop related to nucleation and $3 \mathrm{~d}$ growth ${ }^{15}$ when the potential sweep is reversed (Figure 1b), followed by the anodic stripping peak of the gold deposit from $\mathrm{HOPG}$ at $1.05 \mathrm{~V}$. Therefore, electrochemical data indicate that the kinetics of gold electrodeposition on HOPG can be described as a nucleation and $3 d$ growth process controlled by the reactant diffusion from the solution side.

(14) Sillén, L. G. Stability Constant of Metal-Ion Complexes. Section 1: Inorganic Ligands, The Chemical Society: Burlington House, London, 1964; p 288.

(15) Hernández Creus, A.; Carro, P.; González, S.; Salvarezza, R. C.; Arvia, A. J. Electrochim. Acta 1992, 37, 2215. 
The potentiostatic current transients ( $j$ vs $t$ plots) for $E=0.54 \mathrm{~V}$ (Figure 1c) exhibit an initial current decay, which is related to the double-layer charging process, followed by a current hump at $t=7 \mathrm{~s}$ or thereabouts, and finally a continuous current decay (Figure 1c) fitting a $j$ vs $t^{-1 / 2}$ linear relationship (Figure 1e). These features of potentiostatic current transients for gold electrodeposition on HOPG confirm that a nucleation and $3 \mathrm{~d}$ growth process under diffusion control takes place. ${ }^{16}$

On the other hand, current transients run within the range $-0.1 \mathrm{~V}<E<0.50 \mathrm{~V}$ exhibit only the abovementioned continuous current decay (Figure 1d) fitting also the $j$ vs $t^{-1 / 2}$ linear relationship over a relatively large range of time (Figure 1e), except at shorter times because of the double-layer charging process. Accordingly, no clear current humps in current transients are observed, as the humps appear in almost the same short time range as that corresponding to the relaxation of the electrical double layer. Finally, in all cases, for $t>25 \mathrm{~s}$ a steady current value is reached, as should be expected for a steady freeconvective diffusion process (Figure 1e). Accordingly, in the transient current regime a linear $q$ vs $t^{1 / 2}$ relationship is found, whereas for the steady current regime a linear $q$ vs $t$ relationship is obtained, $q$ being the apparent gold electrodeposition charge density obtained by integration of current transients.

From the $j_{\mathrm{L}}$ value and the $\mathrm{AuCl}_{4}{ }^{-}$ion concentration, ${ }^{14}$ $c_{i} \approx 5 \times 10^{-7} \mathrm{~mol} / \mathrm{cm}^{3}$, the value of the diffusion coefficient $\left(D_{i}\right)$ of $\mathrm{AuCl}_{4}{ }^{-}$ions in the solution was estimated from the equation ${ }^{17}$

$$
j_{\mathrm{L}}=z F D_{i} c_{i} / \delta
$$

where $Z$ is the number of electrons transferred, $F$ is Faraday's constant, and $\delta$ is the thickness of the diffusion layer. By taking for the quiescent solution the value $\delta=$ $0.025 \mathrm{~cm}^{17}$ and $z=3$, it results in $D_{i}=9 \times 10^{-6} \mathrm{~cm}^{2} / \mathrm{s}$, in agreement with values of $D_{i}$ reported in the literature $\left(D_{i}\right.$ $\cong 10^{-5} \mathrm{~cm}^{2} / \mathrm{s}$ ) for the diffusion of complex metal ion in aqueous solutions. ${ }^{18}$

Electrochemical results indicate that, in the range -0.1 $<E<0.5 \mathrm{~V}$ and for $t>1 \mathrm{~s}$, the diffusion zones built up around each growing nucleus overlap and the mass transport process approaches the condition of simple linear diffusion. ${ }^{16}$ For such a situation, the average size of gold aggregates is smaller than $\delta$; i.e., the value of $j$ does not depend on the increasing area of the gold nuclei, as their size is smaller than $\delta$.

3.2. STM Imaging Data. The STM image of a gold electrodeposit grown at $E=0.54 \mathrm{~V}$ (Figure 2a) involving $q=6 \mathrm{mC} / \mathrm{cm}^{2}$ shows the formation of isolated rounded gold crystals $100 \mathrm{~nm}$ in average size which are preferentially nucleated at the step edges of the HOPG. STM images of crystal surfaces taken at the early stages of growth $\left(q=2 \mathrm{mC} / \mathrm{cm}^{2}\right)$ (Figure $\left.2 \mathrm{~b}\right)$ show faceted surfaces and angles between facets which are typical of the $\mathrm{Au}(111)$ face. A large number of kinks at the crystal edges and branches with sharp tips triggered in the direction of HOPG steps can be observed (Figure 2c). The cross section of either sharp tips or kinked edges reveals smooth terrace domains separated by monoatomic high steps. As the electrodeposition time is increased both the average radius $\langle r\rangle$ and average height $\langle h\rangle$ of gold aggregates increase according to $\langle r\rangle \propto t^{0.4}$ (Figure 3a) and $\langle h\rangle \propto t^{0.4}$ (Figure $3 \mathrm{~b}$ ) relationships. In this case, round-shaped

(16) Scharifker, B.; Hills, G. Electrochim. Acta 1983, 288, 79

(17) Levich, V. G. Physicochemical Hydrodynamics; Prentice Hall: Englewood Cliffs, NJ, 1962.

(18) Horvath, A. L. Handbook of Aqueous Electrolyte Solutions; Ellis Horwood: New York, 1985; p 290. $\mathbf{a}$

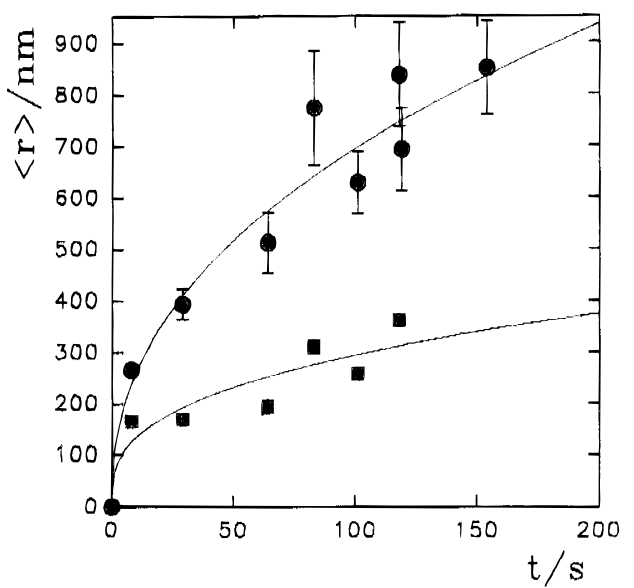

b

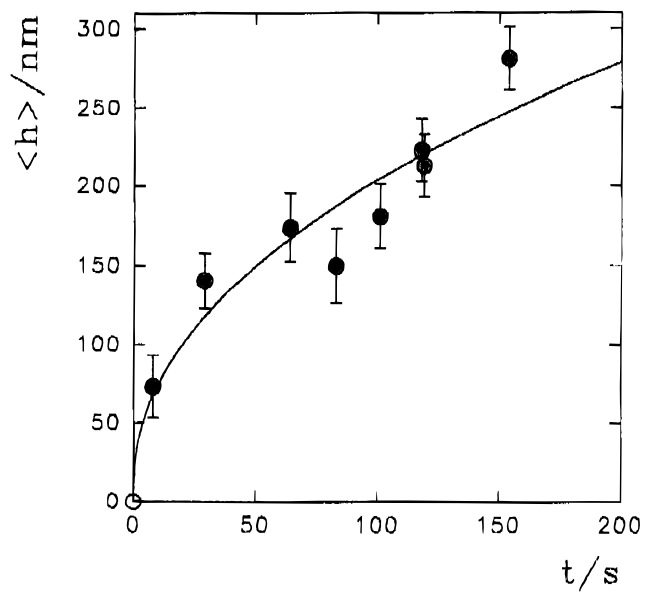

Figure 3. (a) $\langle r\rangle$ vs $t(\bullet)$ and $r_{\mathrm{h}}$ vs $t$ (ם) plots. The upper solid line represents $\langle r\rangle$ vs $t^{2 / 5}$. (b) $\langle h\rangle$ vs $t$ plot for gold aggregates electroformed at $E=0.54 \mathrm{~V}$ and $25^{\circ} \mathrm{C}$. The solid line corresponds to the $h$ vs $t^{2 / 5}$ relationship.

aggregates are formed with the ratio $\langle h\rangle /\langle r\rangle \approx 0.3$. It is evident that when $E=0.54$, tip and branch structures are unstable so that rounded and kinked aggregates are produced. The change in the radius expected for a growing hemispherical crystal $\left(r_{\mathrm{h}}\right)$ calculated from the average instantaneous charge of each nucleus, $\left\langle q_{\mathrm{n}}\right\rangle=q / N$, where $N$ is the crystal density, always yields a value much smaller than $\langle r\rangle$ (Figure 3a). This result suggests that a fast surface relaxation of the crystal surface or/and extensive capture of $\mathrm{Au}$ adatoms at aggregate borders from the HOPG substrate occurs during electrodeposition. This assumption is supported by the small $\langle h\rangle\langle\langle r\rangle$ ratio of crystals.

The atomic resolution STM imaging at domains between gold nuclei shows the interatomic distance $d=0.246 \mathrm{~nm}$, which is typical of the HOPG surface lattice when it is observed by STM, ${ }^{19}$ providing thus clear evidence of the absence of a complete gold atom monolayer preceding the formation of gold aggregates at HOPG steps. The same behaviour has already been found for silver electrodeposition on the same substrate. ${ }^{20}$

On the other hand, STM images of gold electrodeposits built up at $E=0.40 \mathrm{~V}$ (Figure $2 \mathrm{~d})\left(q=6 \mathrm{mC} / \mathrm{cm}^{2}\right)$ show a larger number of uniform irregularly shaped aggregates, their average size being smaller than the size of those

(19) Wiesendanger, R.; Anselmetti, D. In Scanning Tunneling Microscopy I; Guntherodt, H.-J., Wiesendanger, R., Eds.; SpringerVerlag: Berlin, 1992; p 131.

(20) Vázquez, L.; Hernández Creus, A.; Carro, P.; Ocón, P.; Herrasti, P.; Palacio, C.; Vara, J. M.; Salvarezza, R. C.; Arvia, A. J. J. Phys. Chem. 1992, 96, 10454. 
a

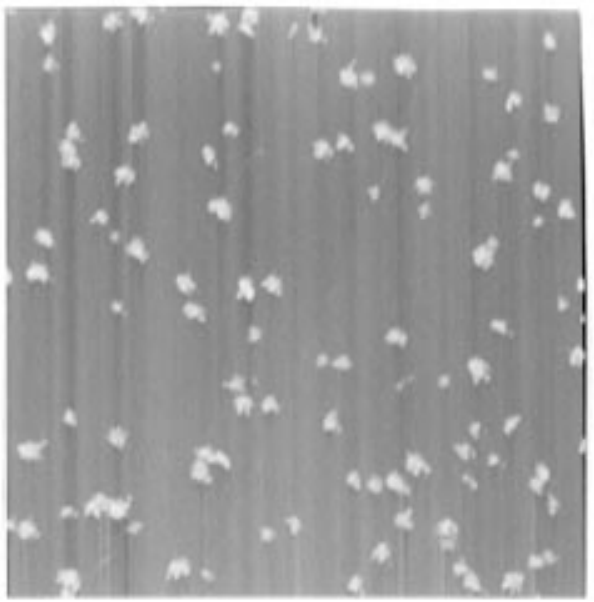

b

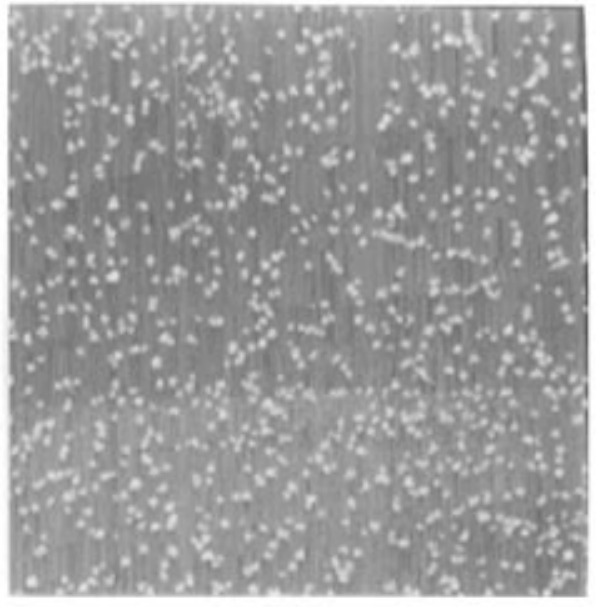

d

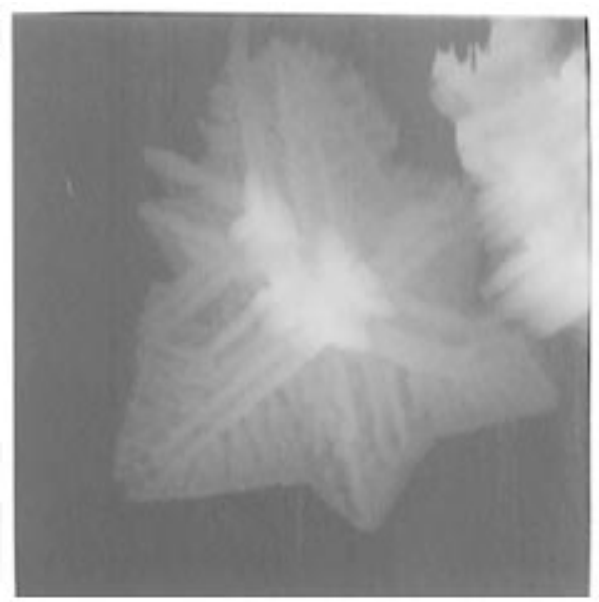

$\mathbf{e}$

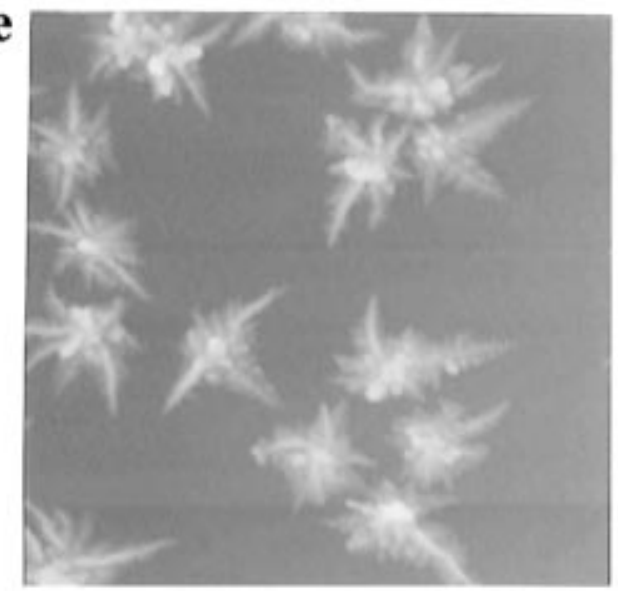

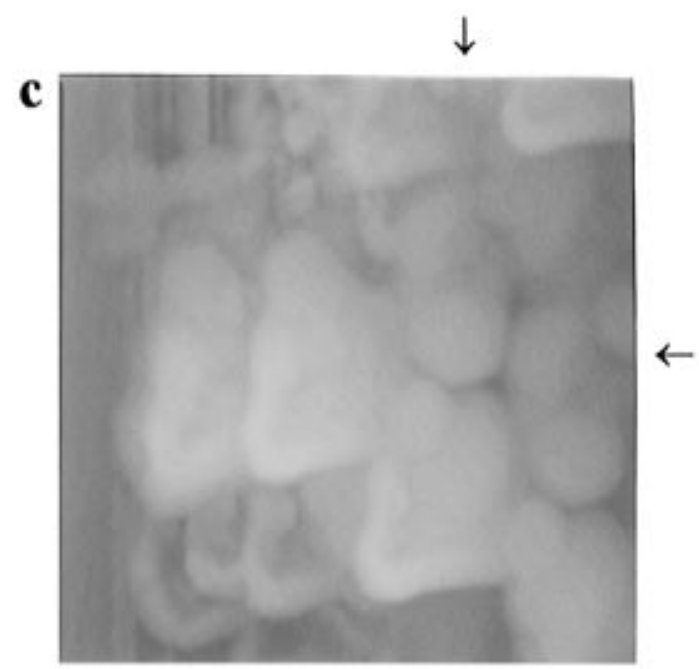

Figure 4. STM images (top view) for gold electrodeposits grown at $E<0.30 \mathrm{~V}$ at $25^{\circ} \mathrm{C}$ : (a) $10 \times 10 \mu \mathrm{m}^{2}, E=0.20 \mathrm{~V}, q=6 \mathrm{mC} / \mathrm{cm}^{2}$; (b) $10 \times 10 \mu \mathrm{m}^{2}, E=0 \mathrm{~V}, q=6 \mathrm{mC} / \mathrm{cm}^{2}$; (c) $0.3 \times 0.3 \mu \mathrm{m}^{2}, E=-0.10 \mathrm{~V}, q=2 \mathrm{mC} / \mathrm{cm}^{2}$ (The triggering of dendrite tips can be observed.); (d) $0.43 \times 0.43 \mu \mathrm{m}^{2}, E=-0.10 \mathrm{~V}, q=6 \mathrm{mC} / \mathrm{cm}^{2}$; (e) $2.2 \times 2.2 \mu \mathrm{m}^{2}, E=-0.10 \mathrm{~V}, q=8 \mathrm{mC} / \mathrm{cm}^{2}$.

formed at $E=0.54 \mathrm{~V}$. For a constant electrodeposition charge $\left(q=6 \mathrm{mC} / \mathrm{cm}^{2}\right)$, as $E$ is shifted from $0.2 \mathrm{~V}$ to -0.1 $\mathrm{V}$, STM images (Figure $4 \mathrm{a}$ and $\mathrm{b}$ ) show that the value of $N$ and the trend to produce dendritic-type morphologies increase (Figure $4 \mathrm{c}-\mathrm{e}$ ). The increase in $N$ becomes more evident from the analysis of both the $N$ vs $E$ and $\langle r\rangle$ vs $E$ plots (Figure $5 \mathrm{a}$ and $\mathrm{b}$ ). The strong dependence of $N$ on $E$ arises from the dependence of the nucleation rate on the oversaturation which is determined by $E .^{21}$ It should be noted that in the range $-0.1 \mathrm{~V}<E<0.3 \mathrm{~V}$ preferred nucleation continues to be seen at step edges, but the number of nuclei formed at terraces begins to increase remarkably.

In the range of potential $-0.1 \mathrm{~V}<E<0.3 \mathrm{~V}$, the aggregate changes from a quasi-spherical shape to a morphology which is characterized by a relatively thick backbone with a large number of thin oriented branches, approaching a dendritic pattern (Figure 4c-e). The evolution of these patterns can be followed at different

(21) Budevski, E. B. In Comprehensive Treatise of Electrochemistry, Conway, B. E., Bockris, J. O'M., Yeager, E., Khan, S. U. M., White, R. M., Eds.; Plenum Press: New York, 1983; Vol. 7, p 399. 

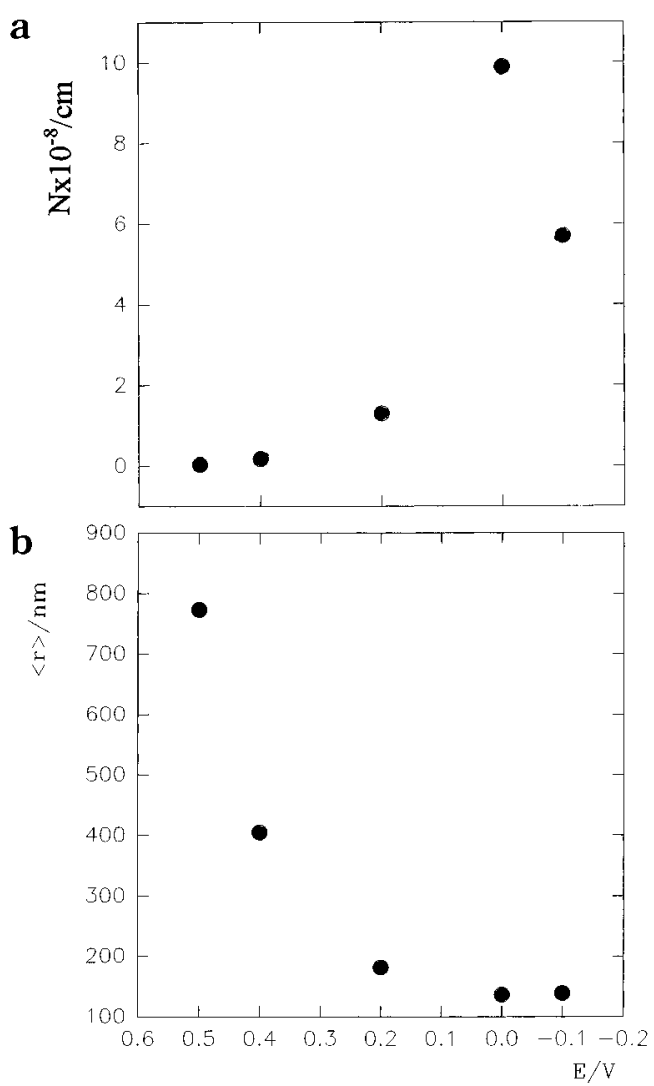

Figure 5. (a) $N$ vs $E$ plot and (b) $\langle r\rangle$ vs $E$ plot for a constant gold electrodeposition charge $\left(q=6 \mathrm{mC} / \mathrm{cm}^{2}\right)$ at $25^{\circ} \mathrm{C}$.

electrodeposition times. Thus, for $E=-0.1 \mathrm{~V}$ and $q=2$ $\mathrm{mC} / \mathrm{cm}^{2}$, initially it results in 3D aggregates forming small hexagonal crystals $10 \mathrm{~nm}$ in average size (Figure 4c, see arrows). Some of these crystals show the appearance of tips which are triggered in certain preferred directions, yielding triangular patterns (Figure 4c). Otherwise, when the same run is extended to $q=6 \mathrm{mC} / \mathrm{cm}^{2}$, tip growth leads to starlike crystalline aggregates (Figure $4 \mathrm{~d}$ ). These aggregates are $300 \mathrm{~nm}$ in average size, and they exhibit a $120^{\circ}$ angle between the principal arm directions and a $60^{\circ}$ angle between the small branch and principal arm directions. Finally, for $q=8 \mathrm{mC} / \mathrm{cm}^{2}$ gold aggregates develop a net dendritic morphology (Figure 4e), as some of the initial arms dominate the growth process to become the backbone of dendrites. At this stage crystalline aggregates $500 \mathrm{~nm}$ in average size and $70 \mathrm{~nm}$ in average height are obtained.

The topography of the dendrite involves central 3d aggregates and backbones approaching a quasi-2D pattern (Figure 6a), as can be seen by a $100 \mathrm{~nm}$ long cross section of a dendrite backbone taken from the origin to tip direction (Figure $6 \mathrm{~b}-\mathrm{d}$ ). The backbone height decreases only $2 \mathrm{~nm}$ from the origin to tip with an average slope close to $1.7^{\circ}$. The tip size is close to $10 \mathrm{~nm}$, and its height fluctuates between 1 and 10 atoms. The backbone surface profile indicates a terrace morphology descending in height from the origin to tip front, the step between terraces being only a few atoms in height. From the cross section analysis it can also be concluded that the tip growth involves a front a few atoms thick.

Finally, for $q>10 \mathrm{mC} / \mathrm{cm}^{2}$ the overlapping of dendritic crystals leads to a percolation pattern, ${ }^{1}$ and then the front a few atoms thick related to tip growth is no longer observed. Further addition of material results in the prevalence of a 3D growth mechanism for the entire electrodeposit. This stage of growth is beyond the scope of this work.
From those experiments comprising $E=-0.1 \mathrm{~V}$ and $q$ $<10 \mathrm{mC} / \mathrm{cm}^{2}$ (Figure $7 \mathrm{a}-\mathrm{c}$ ), the kinetics of gold aggregate formation obeys the following relationship (Figure 8$)^{21}$

$$
N=N_{0}[1-\exp (-A t)]
$$

where $N_{0}$ is the number of initially available nucleation sites at the HOPG surface and $A$ is the steady-state nucleation rate per nucleation site. Experimental data for $E=-0.1 \mathrm{~V}$ fulfill eq 2 for $N_{0}=6 \times 10^{8} \mathrm{~cm}^{-2}$ and $A$ $=0.3 \mathrm{~s}^{-1}$. As can be concluded from Figure 8, after $10 \mathrm{~s}$ all sites available for nucleation become gold nuclei $(N \Longrightarrow$ $\left.N_{0}\right)$.

On the other hand, the values of $\langle r\rangle$ and $\langle h\rangle$ increase similarly with $t$ (Figure 9a and b), indicating that a constant $\langle h\rangle\langle\langle r\rangle$ ratio is involved in the process. Thus, for those aggregates grown at $E=-0.1 \mathrm{~V}$, it results in $\langle h\rangle /\langle r\rangle$ $\simeq 0.3$.

As usually made for the growth of irregular aggregates such as dendrites, ${ }^{22}$ the time dependence of $\langle r\rangle$ is given by the time dependence of the size of the largest tip. When $E=-0.1 \mathrm{~V}$ both $\langle r\rangle$ and $\langle h\rangle$ approach $\langle r\rangle$ vs $t^{1 / 4}$ and $\langle h\rangle$ vs $t^{1 / 4}$ relationships (Figure $9 \mathrm{a}$ and $\mathrm{b}$ ). In this case, the value of $r_{\mathrm{h}}$ from the experimental $q / N$ data is again smaller than $\langle r\rangle$ (Figure 9a). But it is worth noting that the value of $r_{\mathrm{h}}$ is close to the value of the radius of the highest central aggregate (Figures 6a and 9).

It is interesting to emphasize that the $\langle r\rangle$ vs $t^{n}$ relationships involving values of the exponent $n$ in the range $1 / 4$ $\leq n \leq 2 / 5$ observed for the growth of gold crystals in the potential range $-0.1 \mathrm{~V} \leq E \leq 0.54 \mathrm{~V}$ have been derived for the phase growth mechanisms with a significant contribution of surface diffusion. ${ }^{23}$

The fractal dimension $D_{\mathrm{s}}$ of gold aggregates was measured by using the area-perimeter method applied to STM images. ${ }^{24}$ Accordingly, the relation between the perimeter $(P)$ and area $(A)$ for a self-similar object is

$$
P=k A^{D_{\mathrm{s}} / 2}
$$

Gold aggregates can be treated as 3D islands which exhibit at each height measured from the substrate plane a different cross section parallel to the substrate plane. To obtain the value of $D_{\mathrm{s}}$, values of $P$ and $A$ were determined from different island cross sections from the top down to a level close to the HOPG surface. The log $P$ vs $\log A$ plots yield straight lines involving $E$-dependent slopes (Figure $10 \mathrm{a}$ and $\mathrm{b})$. According to eq 3 this means that $D_{\mathrm{s}}$ depends on $E$ varying from $D_{\mathrm{s}}=1.2$ for $E>0.3 \mathrm{~V}$ to $D_{\mathrm{s}}=1.6$ for $E<0.3 \mathrm{~V}$ (Figure 10c). It should be noted that the value $D_{\mathrm{s}}=1.6$ is close to that reported for 2D-dendritic-like patterns, ${ }^{22}$ whereas the value $D_{\mathrm{s}}=1.2$ is slightly higher than $D_{\mathrm{s}}=1.0$, a figure which is expected for a Euclidean contour. The value $D_{\mathrm{s}}=1.2$ is related to the existence of kinked crystal edges and some small unstable branches. Therefore, it can be concluded that the applied potential determines whether a Euclidean-like or a fractal-like pattern will prevail in the formation of gold aggregates on HOPG; i.e., there is a transition in the growth mode which can be controlled by properly adjusting the value of $E$. This conclusion is derived from those patterns of gold aggregates which are formed in the range $-0.1 \mathrm{~V} \leq$ $E \leq 0.54 \mathrm{~V}$. It should be noted that, in these cases, the overall electrochemical process is under mass transport kinetic control from the solution side. Therefore, it appears

(22) Meakin, P. In The Fractal Aproach to Heterogeneous Chemistry, Avnir, D., Ed.; J. Wiley \& Sons: New York, 1990; p 131.

(23) Siegert, M.; Plischke, M. Phys. Rev. Lett. 1994, 73, 1517.

(24) Gómez-Rodríguez, J. M.; Baró, A. M.; Vázquez, L.; Salvarezza, R. C.; Vara, J. M.; Arvia, A. J. J. Phys. Chem. 1992, 96, 347. 

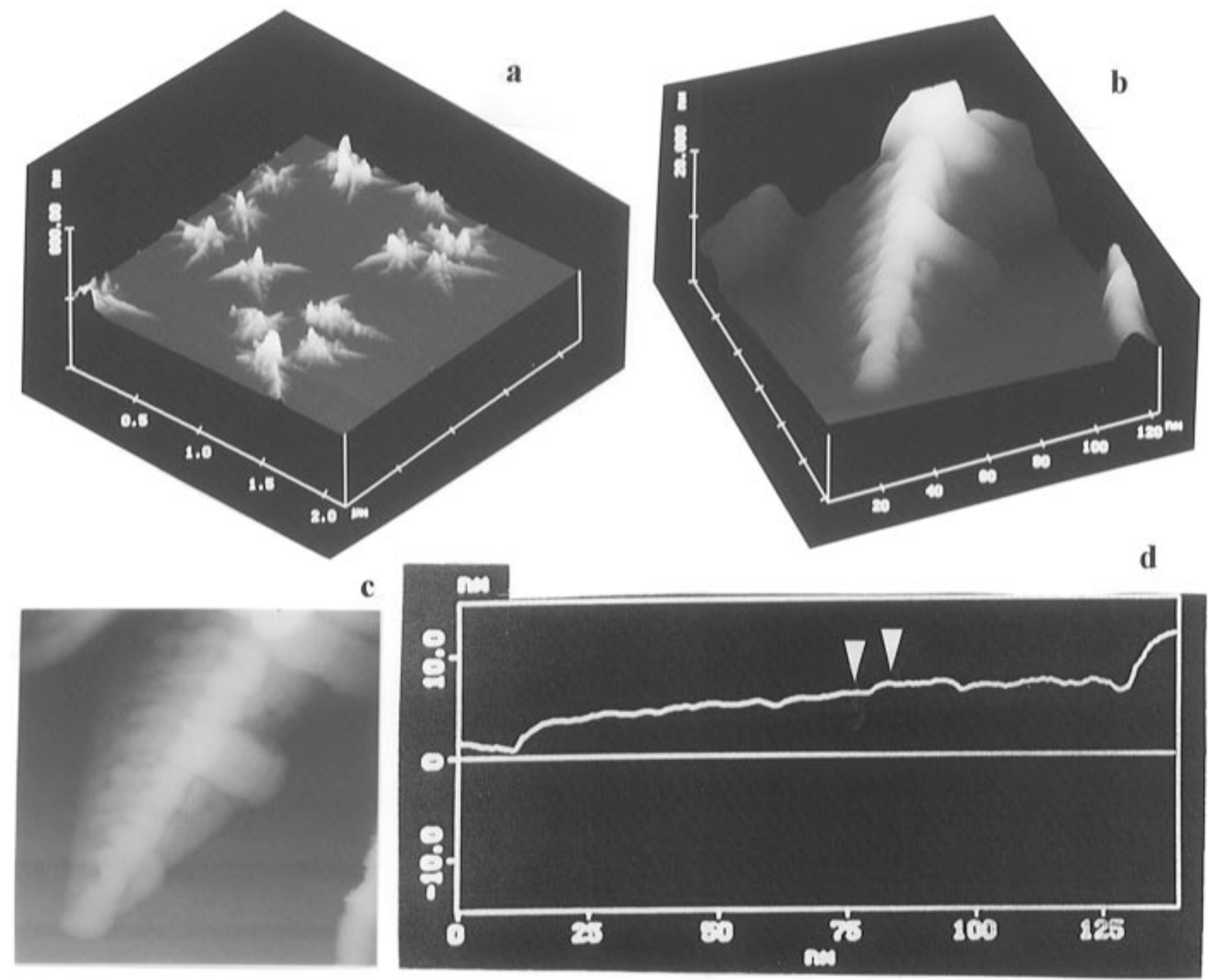

Figure 6. STM images of gold electrodeposit dendritic patterns $\left(q=6 \mathrm{mC} / \mathrm{cm}^{2}\right)$ grown at $E=-0.1 \mathrm{~V}$ and $25{ }^{\circ} \mathrm{C}$ : (a) $2.15 \times 2.15$ $\mu \mathrm{m}^{2}$, 3D image; (b) $0.12 \times 0.12 \mu \mathrm{m}^{2}$, 3D image; (c) $0.12 \times 0.12 \mu \mathrm{m}^{2}$, top view image; (d) cross section of the dendrite backbone shown in part c. A two gold atom high step and smooth terraces can be observed.

that the influence of $E$ on the growth mode of gold aggregates does not involve a significant change in the flux of arriving particles but presumably manifests itself at the level of surface diffusion of gold atoms.

\section{Discussion}

4.1. Possible Gold Electrodeposition Processes. The electrodeposition of gold on HOPG from the plating bath used in this work comprises different growth modes and morphology transitions which depend on the applied potential for gold aggregates formed at step edges and terraces.

In general, STM images at the early stages of growth reveal that $3 \mathrm{D}$ quasi-spherical $\mathrm{Au}(111)$ nuclei are produced by the electroreduction of $\mathrm{AuCl}_{4}^{-}$ions at either edge or terrace preferred nucleation sites, a process which can be represented as follows

$$
\mathrm{AuCl}_{4}^{-}+\mathrm{HOPG}^{*}+3 \mathrm{e}^{-} \Longrightarrow[\mathrm{Au}] \mathrm{HOPG}^{*}+4 \mathrm{Cl}^{-}
$$

where the asterisk denotes a nucleation site at either a step or a HOPG terrace and brackets stand for a gold nucleus. Further electroreduction of $\mathrm{AuCl}_{4}^{-}$ions contributes to an increase in the aggregate size through a global reaction such as

$$
\begin{aligned}
\mathrm{AuCl}_{4}{ }^{-}+[n \mathrm{Au}] \mathrm{HOPG}^{*}+3 \mathrm{e}^{-} \Longrightarrow \\
{[(n+1) \mathrm{Au}] \mathrm{HOPG}^{*}+4 \mathrm{Cl}^{-} }
\end{aligned}
$$

According to electrochemical data (Figures 1c), under the conditions of this work reactions $4 \mathrm{a}$ and $4 \mathrm{~b}$ obey a nucleation and 3D growth under mass transport kinetic control, leading simultaneously to gold nuclei formation, accumulation of $\mathrm{Cl}^{-}$ions at the growing interface, and depletion of $\mathrm{AuCl}_{4}{ }^{-}$ion concentration around the growing nucleus.

Global reactions $4 \mathrm{a}$ and $4 \mathrm{~b}$ correspond to complex electrochemical processes in which the formation of gold adatoms and their surface diffusion on either the HOPG or gold nucleus surface have to be considered, depending whether the electrochemical discharge occurs on either HOPG or gold aggregate surfaces, respectively. In the first case, gold adatoms may form new nuclei on HOPG at preferred sites located at either steps or terraces or they may be added by surface diffusion to edges of existing gold nuclei, promoting a $2 \mathrm{D}$ nucleus growth. In the second case, Au adatoms may move down through Au steps to nucleus edges, leading to 2D growth, or may be trapped on gold terrace sites, promoting a nucleation and 3D growth. 
a

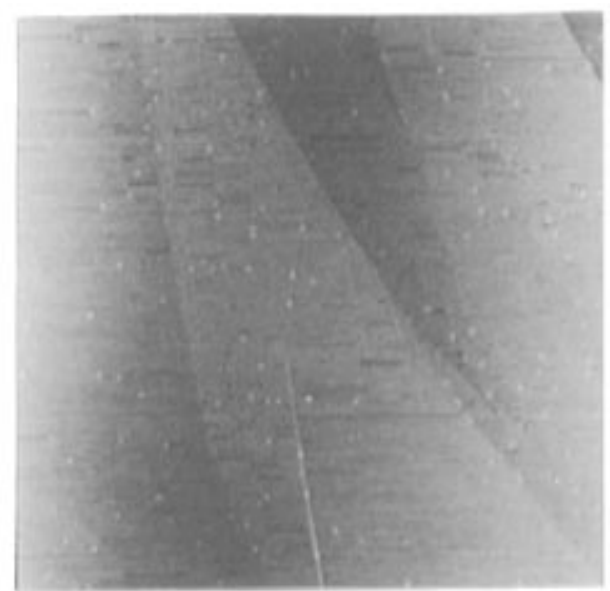

b

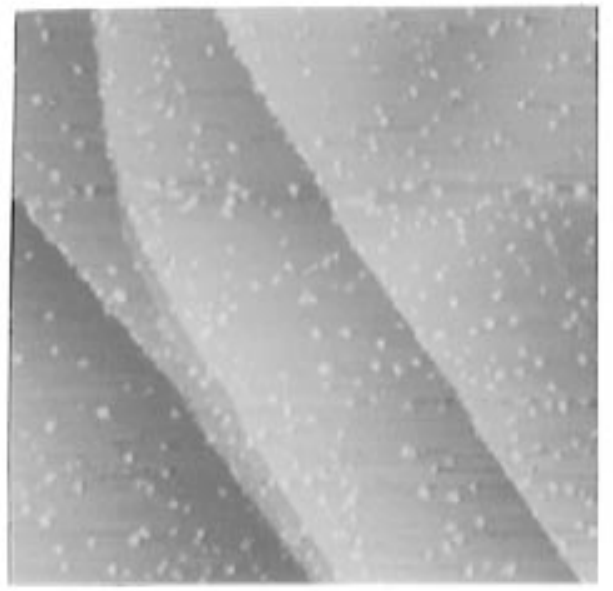

c

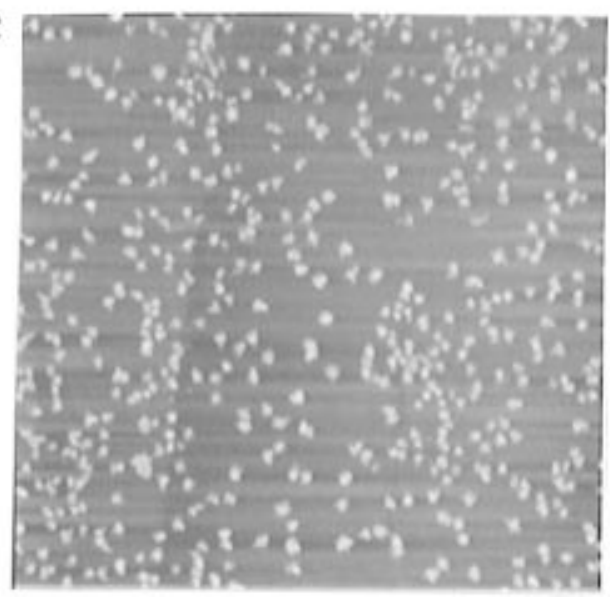

Figure 7. STM images $\left(10 \times 10 \mu \mathrm{m}^{2}\right.$, top view) for gold electrodeposits grown at $E=-0.10 \mathrm{~V}$ at $25^{\circ} \mathrm{C}$ : (a) $q=0.6$ $\mathrm{mC} / \mathrm{cm}^{2}$; (b) $q=4 \mathrm{mC} / \mathrm{cm}^{2}$; (c) $q=6 \mathrm{mC} / \mathrm{cm}^{2}$.

In principle, the discharge of $\mathrm{AuCl}_{4}{ }^{-}$species on HOPG terrace domains might occur as the applied cathodic potential approaches $E_{\mathrm{r}}$, as, in this case, the thickness of the $\mathrm{AuCl}_{4}{ }^{-}$ion-depleted zone around gold nuclei is smaller than the distance between gold nuclei. It has been reported $^{25}$ that for nuclei growing on a solid substrate, at the distance $2 r$, where $r$ is the nucleus radius, the concentration of the electroactive species is close to half its value in the bulk. However, the fact that a small number of large rounded crystals is formed suggests that even when the applied potential approaches $E_{\mathrm{r}}, \mathrm{AuCl}_{4}{ }^{-}$ species are preferentially discharged on gold nuclei rather than on HOPG. This fact becomes clearer as the applied

(25) Fletcher, S. J. Chem. Soc. Faraday Trans., 1 1983, 79, 467.

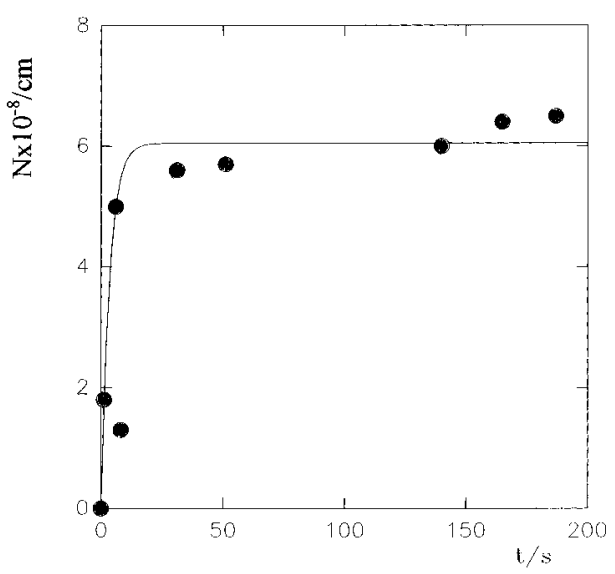

Figure 8. $N$ vs $t$ plot for gold electrodeposits grown at $E=$ $-0.1 \mathrm{~V} ; q<10 \mathrm{mC} / \mathrm{cm}^{2}$. The solid line was calculated with eq 2 taking $N_{0}=6 \times 10^{8} \mathrm{~cm}^{2}$ and $A=0.3 \mathrm{~s}^{-1}$.
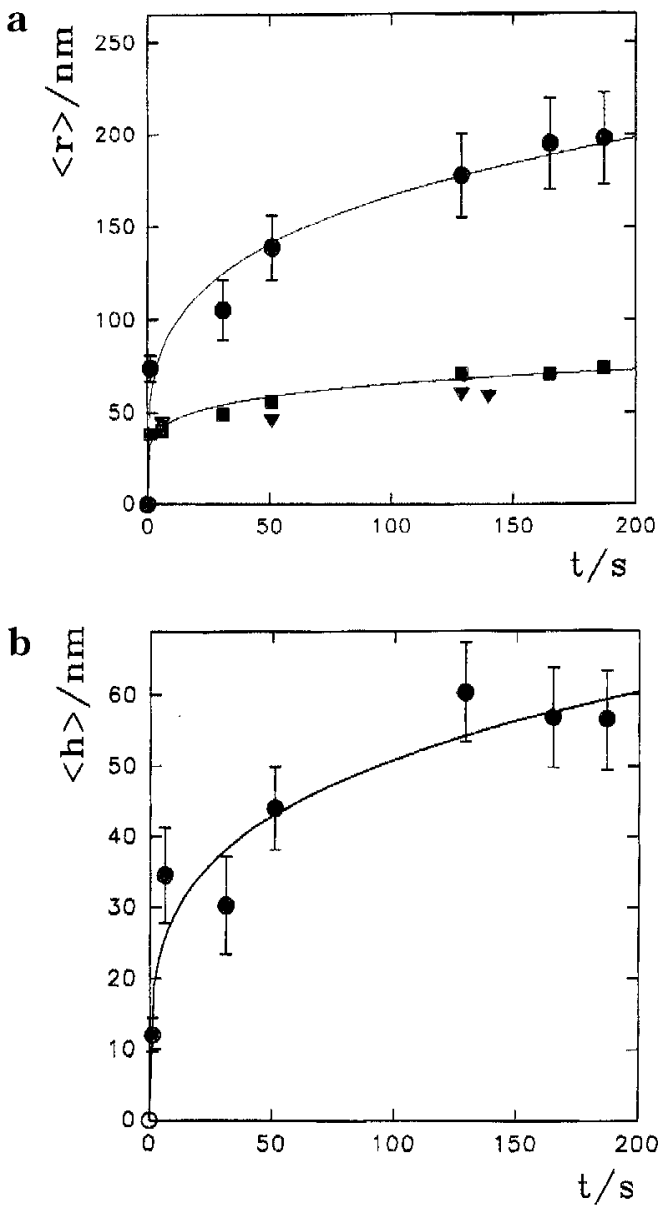

Figure 9. (a) $\langle r\rangle$ vs $t$ plots (๑) derived from STM images. The upper solid line represents the $\langle r\rangle$ vs $t^{1 / 4}$ relationship. (ם) $r_{\mathrm{h}}$ data; $(\boldsymbol{\nabla})\langle r\rangle$ data measured from STM images at the central emerging part of gold dendritic crystals. (b) $\langle h\rangle$ vs $t$ plots derived from STM images. The solid line corresponds to the $h$ vs $t^{1 / 4}$ relationship.

cathodic potential is far from $E_{\mathrm{r}}$. In this case the distance separating gold nuclei becomes small, and then the overlapping of diffusion zones built up around growing nuclei takes place. Consequently, the gold nuclei collect almost all $\mathrm{AuCl}_{4}{ }^{-}$discharging ions, and then practically no electrochemical reaction occurs at bare HOPG domains because the condition $c_{i} \rightarrow 0$ is also approached there.

4.2. Gold Atom Surface Diffusion and Shape of Aggregates. The fact that the average aggregate radius always exceeds the radius calculated for a hemispherical 


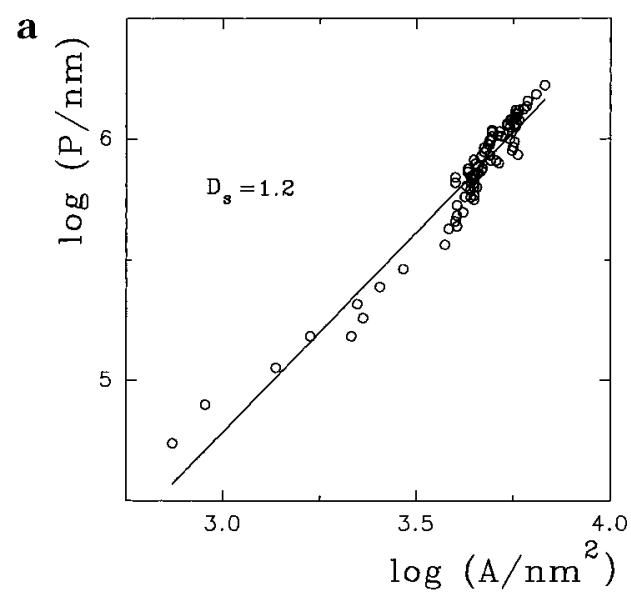

b
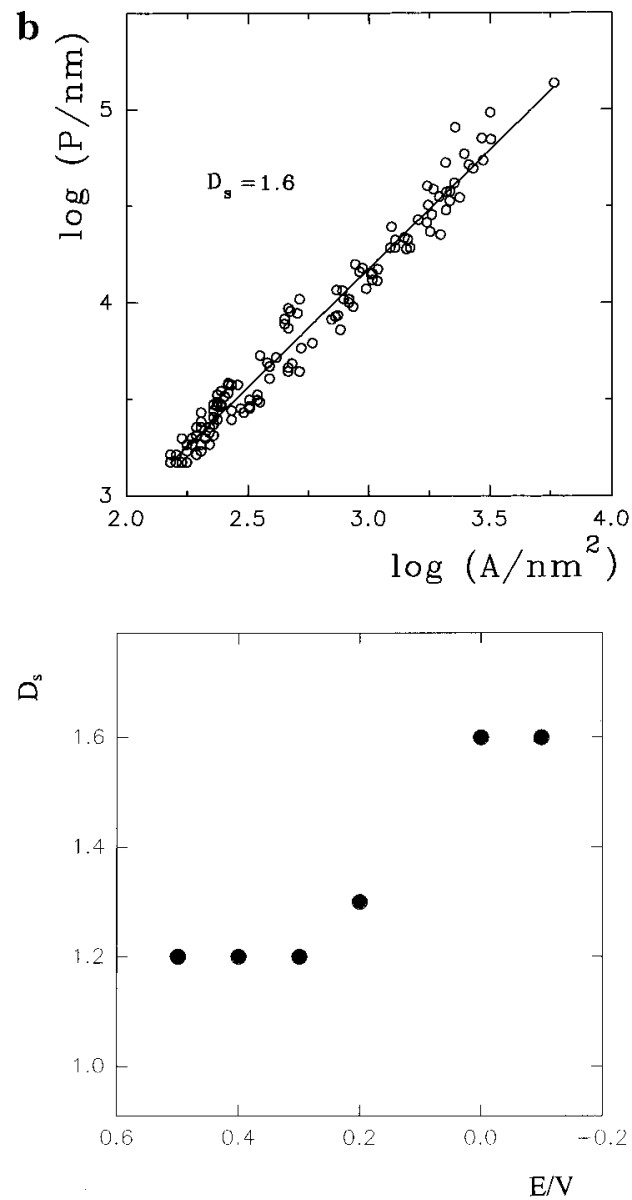

Figure 10. $\log P$ vs $\log A$ plots for gold crystals formed at different values of $E$ : (a) $E=0.54 \mathrm{~V}$; (b) $E=-0.10 \mathrm{~V}$; (c) $D_{\mathrm{s}}$ vs $E$ plot.

aggregate involving the same deposited amount of gold, and the low value of the $\langle h\rangle /\langle r\rangle$ ratio for the crystal shape are indications that a relatively fast surface atom relaxation takes place during aggregate growth. Further considering that this type of relaxation occurs at a relatively low temperature, it seems reasonable to explain the surface relaxation process by a gold adatom surface diffusion mechanism.

After gold nuclei have attained a certain scale size, a change in the growth mode is observed. Thus, the initial quasi-spherical nucleus continues growing isotropically or turns into a dendritic-type aggregate, depending on the applied potential. This transition from the initial spherical nucleus to a dendritic aggregate can be observed when $E<0.3 \mathrm{~V}$. In this case, a stable situation for tip growth is attained and linear $\log \langle r\rangle$ vs $\log t$ and $\log \langle h\rangle$ vs $\log t$ growth laws with the slope $n=1 / 4$ are fulfilled. Otherwise, as the applied potential is changed in the positive direction $(E>0.3 \mathrm{~V})$ the aggregate tends to develop a faceted and kinked surface approaching a Euclidean object. In this case, the same type of kinetic relationship is found with $n=2 / 5$. Both values of $n$ are close to those predicted for growth processes with a significant contribution of surface diffusion. ${ }^{23}$ These facts indicate that gold aggregates grow mainly by collecting Au adatoms arriving by surface diffusion.

4.3. Likely Mechanisms for Gold Crystal Growth. Let us consider two possible mechanisms (I and II) for crystal growth in which gold atom surface diffusion is involved.

In mechanism I gold adatoms produced by the discharge of $\mathrm{AuCl}_{4}{ }^{-}$ions on $\mathrm{HOPG}$ diffuse toward growing gold nuclei to be captured at crystal edges while Au adatoms produced at the top of gold nuclei diffuse down to be attached at crystal edges without restrictions, i.e. in the absence of energy barriers for the surface diffusion of adatoms at step edges as is the case for the deposition, diffusion, and aggregation (DDA) model. ${ }^{1}$ When a bump is formed at a crystal edge, it has a greater chance for capturing arriving gold adatoms rather than a reentrant; therefore, the tip growth is favored. ${ }^{1}$ The DDA model was used to explain the growth of branched gold islands on $\mathrm{Ru}(0001) .^{1,26}$

In principle, at cathodic potentials close to $E_{\mathrm{r}}$ mechanism I would be operative as the direct discharge of $\mathrm{AuCl}_{4}{ }^{-}$ species on HOPG also becomes possible. In this case, if mechanism I operates, patterns such as those derived from the DDA model will be expected. For this model tip splitting rather than tip stabilization becomes dominant. Certainly, dendritic patterns could also be produced from the DLA model if the noise reduction technique is included. ${ }^{22}$ However, the rounded and kinked crystals which are formed in our experiments at cathodic potentials close to $E_{\mathrm{r}}$ are far from either DLA-like or dendritic patterns, as tips are unstable. Otherwise, it was found that, by increasing the substrate temperature, the surface diffusion of atoms at crystal edges is enhanced and this results in a transition from DLA-like to rounded compact and kinked islands. ${ }^{1}$ Hence, our results for $E \geq 0.3 \mathrm{~V}$ would support the idea that gold atom diffusion at crystal edges plays a relevant role in eliminating either the DLA or dendritic-like structure. The possible origin of this enhancement of surface diffusion at crystal edges in electrochemically grown gold aggregates will be discussed further on.

Another drawback in explaning our results through a simple DDA model lies in the fact that this model predicts perfect 2D patterns because adatoms on top of a nucleus move down without restriction to reach crystal edges. The experimental value $\langle h\rangle /\langle r\rangle \simeq 0.3$ observed in the overall $E$ range indicates that gold adatoms produced by the discharge of $\mathrm{AuCl}_{4}{ }^{-}$species on the aggregate top encounter an energy barrier at gold crystal steps. ${ }^{27}$ For a barrierless surface diffusion the development of a completely flat interface similar to that predicted by the EdwardsWilkinson model ${ }^{28}$ should be expected. It has been reported that the energy barrier for a gold atom diffusing down from an island is larger than that for an atom diffusing on a flat terrace. ${ }^{29,30}$ Therefore, it is reasonable to assume that barriers for surface atom diffusion also

(26) Hwang, R. Q.; Schroder, J.; Gunther, G.; Behm, R. J. Phys. Rev. Lett. 1991, 67, 3279 .

(27) Schwoebel, R. J. Appl. Phys. 1969, 40, 614

(28) Edwards, S. F.; Wilkinson, D. R. Proc. R. Soc. London 1982, A381, 17 .

(29) Erlich, G. Surf. Sci. 1994, 299/300, 628 and references therein.

(30) Li, Y.; DePristo, E. Surf. Sci. 1996, 351, 189. 

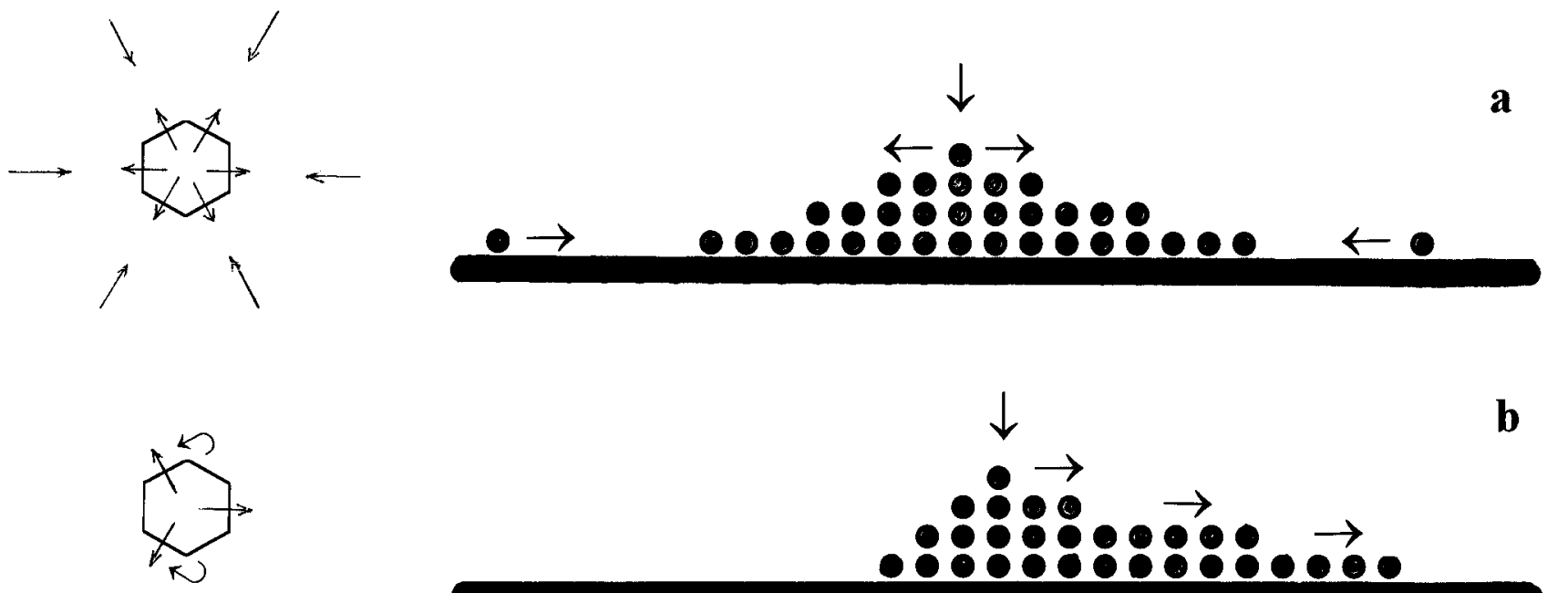

b

Figure 11. 2D schemes of gold surface atom motion on HOPG related to growth modes at (a) $E>0.3 \mathrm{~V}$ and (b) $E<0.3 \mathrm{~V}$. Black points denote gold atoms; black stripes stand for the HOPG surface profile; arrows indicate preferred directions for the surface diffusion of gold atoms. Curved arrows in scheme b indicate the corner diffusion promoted by the anisotropic energy barrier for surface diffusion at edges.

play a relevant role in the gold crystal growth mode. It should be noted that when an isotropic barrier is considered, the growing aggregate should evolve, maintaining its original either rounded or hexagonal shape. The magnitude of the barrier acting at step edges is closely related to the coarsening exponent $n$. Values of $n$ of the order of $2 / 5$ have been observed for a relatively weak stepedge barrier. ${ }^{31}$

From the above discussion it can be concluded that mechanism I cannot explain the appearance of kinked and rounded crystals with $\langle h\rangle\langle\langle r\rangle \simeq 0.3$ observed at $E>$ $0.3 \mathrm{~V}$, which result from both the existence of an isotropic weak-energy barrier at steps for descending Au adatoms, promoting 3D growth, and the remarkable contribution of edge diffusion, assisting the elimination of those instabilities which lead to DLA and dendritic patterns. A scheme showing the isotropic surface diffusion of gold atoms for crystal growth at $E>0.3 \mathrm{~V}$ is shown in Figure 11 a.

Mechanism II for crystal growth focuses mainly on the role played by gold adatoms formed by discharge on gold coupled with the existence of an anisotropic energy barrier at gold crystal step edges. As shown by the experimental results, this situation becomes more relevant for $E<0.3$ $\mathrm{V}$; due to the small average internucleus distance, nuclei capture almost all arriving $\mathrm{AuCl}_{4}{ }^{-}$species. The value of $n=1 / 4$ for the aggregates growing at $E<0.3 \mathrm{~V}$ indicates a stronger step-edge barrier than that observed for the aggregates grown at $E>0.3 \mathrm{~V}$. Dendritic patterns observed in this potential range indicate the presence of an anisotropic energy barrier at steps in contrast to the isotropic energy barrier which explains aggregate formation for $E>0.3 \mathrm{~V}$. An anisotropic energy barrier should produce a change from either a rounded or a hexagonal shape to either a starlike ${ }^{32}$ or a dendritic-like growth pattern. ${ }^{33}$ A scheme showing the anisotropic surface diffusion of gold adatoms for crystal growth at $E<0.3 \mathrm{~V}$ is shown in Figure $11 \mathrm{~b}$. It should be noted that for $\mathrm{Au}(111)$, at potentials close to $E_{\mathrm{pzc}}$, the potential of zero charge, the transition from the unreconstructured $(1 \times 1)$ structure of $\mathrm{Au}(111)$ to the striped phase consisting of the

(31) Smilauer, P.; Vvedenski, D. D. Phys. Rev. B 1995, 52, 14263.

(32) Jacobsen, J.; Jacobsen, K. W.; Stoltze, S.; Norskov, J. K. Phys. Rev. Lett. 1995, 74, 2295.

(33) Brune, H.; Roder, H.; Bromann, K.; Kern, K.; Jacobsen, J.; Stoltze, P.; Jacobsen, K.; Norskov, J. Surf. Sci. 1996, 349, L115 and references therein. reconstructed $(p \times \sqrt{ } 3)$ structure takes place. ${ }^{34}$ Then, hexagonalgold crystals are preferentially formed at negative potentials in order to lower the surface energy. ${ }^{35}$

From the atomistic point of view it is possible to distinguish two diffusion processes that can take place at crystal edges depending on whether an adatom starts from a site which is laterally two- (edge diffusion) or onefold coordinated to the crystal edge (corner diffusion). ${ }^{33}$ Dendritic patterns with trigonal symmetry on hexagonal surfaces have been generated, introducing anisotropic corner surface atom diffusion, whereas edge diffusion leads mainly to a thickening of the aggregate's branches. It should also be noted that the specific substrate symmetry plays a relevant role in the development of dendritic patterns. ${ }^{33}$ This fact can explain why no dendritic crystals were observed during the gold electrodeposition onto amorphous carbon in a similar electrolyte and under similar experimental conditions. ${ }^{35}$

4.4. Applied Electric Potential Dependence of Gold Atom Surface Diffusion. On the basis of the preceding discussion, results shown in this work point out that the energy barrier for adatom diffusion at step edges becomes potential dependent, and it turns out to change from isotropic to anisotropic as the potential is moved from the $E>0.3 \mathrm{~V}$ to the $E<0.3 \mathrm{~V}$ region. This change implies that, for $E<0.3 \mathrm{~V}$, the energy barrier height at the edge of each crystal becomes higher at certain step directions as compared to others, favoring thus the dendritic growth by triggering a stable tip in the step direction where the lowest energy barrier is found. The decrease in the coarsening exponent from $n=2 / 5$ to $1 / 4$ as $E$ is moved negatively is a clear indication that the height of the energy barrier at certain step directions has been increased. ${ }^{31}$

A possible origin of the potential-dependent crystal shape is $\mathrm{Cl}^{-}$anion adsorption on gold, as these ions are not only present in the electrolyte solution but also accumulate at the metal/solution interface during the electrochemical reaction. In fact, for $E<0.3 \mathrm{~V}$, a Au(111) single crystal in contact with the electrolyte solution should be negatively charged, as the potential of zero charge for

(34) Wandlowski, Th.; Ocko, B. M.; Magnussen, O. M.; Wu, S.; Lipkowski, J. J. Electroanal. Chem. 1996, 409, 155.

(35) Lu, D.-I.; Okawa, Y.; Ichihara, M.; Aramata, A.; Tanaka, K.-I J. Electroanal. Chem. 1996, 406, 101. 
$\mathrm{Au}(111)$ is $E_{\mathrm{pzc}}=0.30 \mathrm{~V}$ at $25^{\circ} \mathrm{C} .{ }^{36}$ Then, for $E<E_{\mathrm{pzc}}$ no marked influence of $\mathrm{Cl}^{-}$anion adsorption would be expected, so that the behavior of the system would approach that observed for the growth of dendritic Ag crystals on $\mathrm{Pt}(111)$ in vacuum, where anisotropic corner diffusion operates. ${ }^{33}$ Conversely, at $E>E_{\mathrm{pzc}}$, the gold surface is positively charged and a substantial influence of $\mathrm{Cl}^{-}$anion adsorption should be expected. It is wellknown that for gold single crystal surfaces the potentialdependent $\mathrm{Cl}^{-}$anion adsorption enhances adatom mass transport ${ }^{37}$ and step movement. ${ }^{38}$ It has been reported that the adsorption of $\mathrm{Cl}^{-}$ions changes the surface diffusion coefficient of $\mathrm{Au}$ adatoms on gold immersed in an acid solution from $1 \times 10^{-12} \mathrm{~cm}^{2} / \mathrm{s}$ to $8 \times 10^{-12} \mathrm{~cm}^{2} / \mathrm{s}$ when $E$ is increased from $0.30 \mathrm{~V}$ to $0.80 \mathrm{~V} .^{39}$ Hence, the adsorption of $\mathrm{Cl}^{-}$anion at step edges produces a decrease in the height of the surface adatom diffusion energy barrier approaching isotropic surface diffusion conditions, which favors the growth of kinked rounded crystals. This explanation is supported by the change from $n=2 / 5$ to $n$ $=1 / 4$ and by the change from $D_{\mathrm{s}}=1.6$ for $E<0.3 \mathrm{~V}$ to $D_{\mathrm{s}}$ $=1.2$ for $E>0.3 \mathrm{~V}$ (Figure $10 \mathrm{c}$ ).

Finally, it might be argued that the formation of dendritic gold patterns on HOPG would be similar to that of dendritic copper patterns grown on copper by electrodeposition from $\mathrm{Cl}^{-}$ion-containing acid solution. ${ }^{40}$ In this case, copper electrodeposition has been studied under charge transfer control, and the growth rate depended on the difference $E-E_{\mathrm{pzc}}$ for each crystallographic face. Accordingly, dendritic pattern formation has been explained by the anisotropy in the growth rates of the copper crystallographic faces due to the large differences in the corresponding $E_{\mathrm{pzc}}$ values. The situation for gold dendrite formation $\left(E \ll E_{\mathrm{r}}\right)$ in the presence of a large excess of supporting electrolyte reported in our work is rather different, as it occurs under mass transport kinetic control in the solution, which implies that the electrochemical

(36) Hamelin, A. In Modern Aspects of Electrochemistry; Conway, B. E., White, R. M., Bockris, J. O'M., Eds.; Plenum Press: New York, 1985; Vol. 16, p 1 .

(37) Gao, X.; Edens, G. J.; Weaver, M. J. J. Electroanal. Chem. 1994 376,21 and references therein.

(38) Trevor, D. J.; Chidsey, C. E. D.; Loiacono, D. N. Phys. Rev. Lett. 1989, 62, 929 .

(39) García, M. P.; Gómez, M. M.; Salvarezza, R. C.; Arvia, A. J. J. Electroanal. Chem. 1993, 347, 237. 2980. reaction is less sensitive to surface orientation. ${ }^{40}$ Therefore, it appears that gold dendrite formation is dominated by the anisotropy of gold atom surface diffusion on gold, which depends on $\mathrm{Cl}^{-}$ion adsorption instead of an anisotropic deposition rate on the different crystallographic faces. In addition, preliminary results for gold electrodeposit growth modes on HOPG in solution containing an increasing amount of $\mathrm{Cl}^{-}$ions show a trend to suppress dendritic growth. These results will be extensively discussed in a forthcoming publication. ${ }^{41}$

\section{Conclusions}

The kinetics of gold electrodeposition on HOPG from aqueous $\mathrm{Cl}^{-}$-containing acid solution proceeds by a nucleation and 3D growth mechanism under mass transport control.

The electrodeposition of gold on HOPG involves the formation of gold adatoms on both the HOPG surface and gold nuclei. A fast relaxation of gold aggregates occurs during deposition due to the surface diffusion of gold adatoms at crystal edges. The relative contribution of gold adatom diffusion from both HOPG and gold aggregate surfaces changes with the applied potential value.

Gold aggregates increase their fractal dimension as the applied potential is shifted in the negative direction.

Dendritic aggregates are produced when the gold surface is negatively charged $\left(E<E_{\text {pzc }}\right)$. This growth mode results from the anisotropic corner surface diffusion, caused by energy barriers for surface atom diffusion of different heights at step edges, as has been observed for metal deposition from vapor.

Rounded kinked gold crystals are formed when the gold surface is positively charged $E>E_{\mathrm{pzc}}$. This fact results from the leveling and lowering of barrier heights at step edges induced by $\mathrm{Cl}^{-}$anion adsorption on gold aggregates.

Acknowledgment. This work was financially supported by projects IN94-05553 and PB94-0592A (DGICYT, Spain). R.C.S. and A.J.A. thank the Dirección General de Investigación Científica y Enseñanza Superior (Spain) and Consejo Nacional de Investigaciones Científicas y Técnicas (Argentina) for financial support.

\section{LA960700A}

(41) Martìn, H.; Hernàndez Creus, A.; Carro, P.; Salvarezza, R. C.; Arvia, A. J. In preparation. 